Bulletin of the Institute of Mathematics

Academia Sinica (New Series)

Vol. 13 (2018), No. 3, pp. 351-363

DOI: 10.21915 /BIMAS.2018304

\title{
CANONICAL LEFT CELLS AND THE LOWEST TWO-SIDED CELL IN AN AFFINE WEYL GROUP
}

\author{
NANHUA XI \\ Dedicated to Professor George Lusztig on the occasion of his 70th birthday \\ Institute of Mathematics, Chinese Academy of Sciences, Beijing, 100190 and School of Mathematical \\ Sciences, University of Chinese Academy of Sciences, Beijing 100049, China. \\ E-mail: nanhua@math.ac.cn
}

\|\|$\|$

\begin{abstract}
We give some discussions to the relations between canonical left cells and the lowest two-sided cell of an affine Weyl group. In particular, we use the relations to construct irreducible modules attached to the lowest two-sided cell and some one dimensional representations of an affine Hecke algebra.
\end{abstract}

Canonical left cells of an affine Weyl group are interesting in understanding cells in affine Weyl groups and have nice relations with the structure and representations of algebraic groups. However, it is not easy to describe canonical left cells. In this paper we give some discussions to the relations between canonical left cells and the lowest two-sided cell of an affine Weyl group. In particular, we use the relations to construct irreducible modules attached to the lowest two-sided cell (see Theorem4.1) and some one dimensional representations of an affine Hecke algebra (see Theorem 3.5). For convenience we work with an extend affine Weyl group. This work was partially motivated by [1].

Received August 1, 2016 and in revised form December 19, 2016.

AMS Subject Classification: Primary 20C08.

Key words and phrases: Affine Weyl group, canonical left cell, lowest two-sided cell.

This work is partially supported by Natural Sciences Foundation of China (No. 11321101). 


\section{Canonical Left Cells}

1.1. Let $R$ be an irreducible root system and $P$ the corresponding weight lattice. The Weyl group $W_{0}$ acts on $P$ naturally and the semi-direct product $W=W_{0} \ltimes P$ is an extended affine Weyl group, which contains the affine Weyl group $W_{a}=W_{0} \ltimes \mathbb{Z} R$. Let $S$ be the set of simple reflections of $W_{a}$. The partial order $\leq$ and the length function $l$ on $W$ are well defined. The operation on $P$ will be written in multiplication.

For $w \in W$, set $L(w)=\{s \in S \mid s w \leq w\}$ and $R(w)=\{s \in S \mid w s \leq w\}$. Let $s_{0}$ be the unique simple reflection of $W_{a}$ out of $W_{0}$. Define $Y_{0}=\{w \in$ $\left.W \mid R(w) \subseteq\left\{s_{0}\right\}\right\}$. Then $Y_{0} \cap \Omega$ is a left cell for any two-sided cell $\Omega$ of $W$, called a canonical left cell.

In general it is not easy to describe a canonical left cell. However, it is easy to describe the set $Y_{0}$. Let $w_{0}$ be the longest element of $W_{0}$. The set of anti-dominant weights in $P$ is defined to be $P^{-}=\left\{x \in P \mid l\left(x w_{0}\right)=\right.$ $\left.l\left(w_{0}\right)+l(x)\right\}$ and the set of dominant weights is $P^{+}=\left\{x \in P \mid l\left(w_{0} x\right)=\right.$ $\left.l\left(w_{0}\right)+l(x)\right\}$.

Proposition 1.2. $Y_{0}=\left\{w x \mid w \in W_{0}, x \in P^{-}\right.$and $\left.R(w) \subseteq L(x)\right\}$.

Proof. Let $u \in W$. Then there exist unique $w, v \in W_{0}$ and $x \in P^{-}$such that $R(w) \subseteq L(x)$ and $u=w x v$. Moreover, we have $l(u)=l(x)+l(v)-l(w)$. The proposition follows.

1.3. It would be interesting to see when two elements in $Y_{0}$ are in a left cell. Let $\rho$ be the product of all fundamental dominant weights. Then the set $\left\{w x \rho^{-1} \mid w \in W_{0}, x \in P^{-}\right.$and $\left.R(w) \subseteq L(x)\right\}$ is the canonical left cell in the lowest two-sided cell $c_{0}$ of $W$. In general, for any $x \in P^{-}$there exists a positive integer $a$ (depending on $x$ ) such that $x^{b}$ and $x^{a}$ are in a left cell if $b \geq a$ (see [8, Lemma 3.2]). It seems that the number $a$ is small, in many cases, it is among $1,2,3$.

Let $S_{0}=S \cap W_{0}$ and denote by $\Gamma_{0}$ the left cell $\left\{w \in W \mid R(w)=S_{0}\right\}$, which is in the lowest two-sided cell $c_{0}$ of $W$. For $x \in P$, denote by $n_{x}$ (resp. $m_{x}$ ) the unique shortest element in the coset $x W_{0}$ (resp. the double coset $\left.W_{0} x W_{0}\right)$. The map $x \rightarrow n_{x}$ defines a one-to-one correspondence from $P$ to $Y_{0}$, and the map $n_{x} \rightarrow n_{x} w_{0}$ defines a one-to-one correspondence from $Y_{0}$ to $\Gamma_{0}$. Also the map $x \rightarrow m_{x}$ defines a one-to-one correspondence between 
$P^{+}$and $Y_{0} \cap Y_{0}^{-1}$. The sets $Y_{0}$ and $\Gamma_{0}$ produce naturally two modules of an affine Hecke algebra of $(W, S)$. In next section we will see that the two modules are essentially the same.

\section{Cell Modules of Affine Hecke Algebras}

2.1. Let $H$ be the Hecke algebra of $(W, S)$ over a field $k$ with parameter $q$. Assume that $k$ contains square roots of $q$. Let $\left\{T_{w}\right\}_{w \in W}$ be its standard basis. For any $w$ in $W$, let

$$
C_{w}=q^{-\frac{l(w)}{2}} \sum_{y \leq w} P_{y, w}(q) T_{y}
$$

and

$$
C_{w}^{\prime}=q^{\frac{l(w)}{2}} \sum_{y \leq w}(-1)^{l(w)-l(y)} q^{-l(y)} P_{y, w}\left(q^{-1}\right) T_{y},
$$

where $P_{y, w}$ are the Kazhdan-Lusztig polynomials. Then the elements $C_{w}$, $w \in W$, form a basis of $H$, and the elements $C_{w}^{\prime}, w \in W$, form a basis of $H$ as well, see [2].

For any $x \in P$ there is a well defined element $\theta_{x}=q^{-\frac{l(y)}{2}} T_{y} q^{\frac{l(z)}{2}} T_{z}^{-1}$. where $y, z \in P^{+}$such that $x=y z^{-1}$. Then $\theta_{x} \theta_{y}=\theta_{y} \theta_{x}$ for any $x, y \in P$ and the elements $T_{w} \theta_{x}$ (resp. $\left.\theta_{x} T_{w}\right), w \in W_{0}, x \in P$, form a basis of $H$. See [4].

The group algebra $k[P]$ is isomorphic to the subalgebra $\Theta$ of $H$ generated by all $\theta_{x}, x \in P$. Lusztig defined several $H$-module structures on $k[P]$, see [5, Section 7]. They are actually isomorphic to the modules provided by the left cell $\Gamma_{0}$. Let $M$ (resp. $M^{\prime}$ ) be the subspace of $H$ spanned by all $C_{w}, w \in \Gamma_{0}\left(\operatorname{resp} . C_{w}^{\prime}, w \in \Gamma_{0}\right)$. Then $M$ and $M^{\prime}$ are left ideals of $H$ and generated by $C=C_{w_{0}}$ and $C^{\prime}=C_{w_{0}}^{\prime}$ respectively. The elements $\theta_{x} C, x \in P$, form a basis of $M$ and the elements $\theta_{x} C^{\prime}, x \in P$, form a basis of $M^{\prime}$.

Let $\mathcal{I}$ (resp. $\mathcal{I}^{\prime}$ ) be the subspace of $H$ spanned by all $C_{w}, w \in W-Y_{0}$ (resp. $C_{w}^{\prime}, w \in W-Y_{0}$ ). Then $\mathcal{I}$ and $\mathcal{I}^{\prime}$ are left ideals of $H$. Let $N=H / \mathcal{I}$ and $N^{\prime}=H / \mathcal{I}^{\prime}$. Essentially the following result is due to Arkhipov and Bezrukavnikov (see [1, 1.1.1]).

Lemma 2.2. As $H$-modules $N$ is isomorphic to $M^{\prime}$, and $N^{\prime}$ is isomorphic to $M$. 
Proof. Consider the surjective homomorphism $H \rightarrow M^{\prime}, h \rightarrow h C^{\prime}$. It is easy to check that the kernel is $\mathcal{I}$. So $N$ is isomorphic to $M^{\prime}$. Similarly the surjective homomorphism $H \rightarrow M, h \rightarrow h C$ induces an isomorphism $N^{\prime} \rightarrow M$ of $H$-module. The lemma is proved.

2.3. The geometric explanation of the isomorphism in the above lemma is that Thom isomorphism for a certain equivariant $K$-group of the cotangent bundle of flag variety is compatible with certain actions of the affine Hecke algebra $H$, see [5, Section 7].

Lemma 2.2 seems helpful in understanding the structure of $H$-modules $M$ and $M^{\prime}$, and may be useful to understand canonical left cells. A natural question is to consider the submodule of $M^{\prime}$ (resp. $M$ ) generated by all $C_{w} C^{\prime}$ (resp. $\left.C_{w}^{\prime} C\right), w \in c_{0} \cap Y_{0}$. Modulo a central character of $H$, we can get a finite dimensional quotient algebra of $H$. In next two sections we will give some discussion to the images in such quotient algebras of the submodules. We will show that the images in such a quotient algebra is either irreducible $H$-module or zero when $k$ is algebraically closed (Theorem 4.1).

\section{A Realization of Some One Dimensional Representations}

In this section we construct some one dimensional representations of the affine Hecke algebra $H$ through certain quotient algebras of $H$ (see Theorem 3.5).

3.1. From now on we assume that $k$ is algebraically closed. Recall that $\Theta$ is the subalgebra of $H$ generated by all $\theta_{x}, x \in P$. Let the Weyl group $W_{0}$ act on $\Theta$ by $w\left(\theta_{x}\right)=\theta_{w(x)}$.

We shall need several formulas in $H$. Let $x \in P$, the Macdonald formula says (see [6, Theorem 2.22])

$$
C \theta_{x} C=q^{-\frac{l\left(w_{0}\right)}{2}} \sum_{w \in W_{0}} w\left(\theta_{x} \prod_{\alpha \in R^{+}} \frac{1-q \theta_{\alpha}}{1-\theta_{\alpha}}\right) C .
$$

Let $\Delta$ be the set of simple roots of $R$ and denote $x_{\alpha}$ the fundamental dominant weight corresponding to a simple root $\alpha$. Recall that $\rho=x_{\Delta}$ is 
the product of all fundamental dominant weights. Using [4, Corollary 7.8, Lemma 7.4 (iii)] we get

$$
\begin{aligned}
C^{\prime} \theta_{\rho^{-1}} C & =q^{-\frac{\nu}{2}} C^{\prime} \sum_{I \subseteq R^{+}}(-q)^{|I|} \theta_{\rho^{-1}} \theta_{\alpha_{I}}, \\
C^{\prime} \theta_{\rho} C & =q^{\frac{\nu}{2}} C^{\prime} \sum_{I \subseteq R^{+}}(-q)^{-|I|} \theta_{\rho} \theta_{\alpha_{I}^{-1}},
\end{aligned}
$$

where $\nu=l\left(w_{0}\right)=\left|R^{+}\right|, \alpha_{I}$ is the sum of all roots in $I$ and $|I|$ is the cardinality of $I$.

There is a unique involutive anti-automorphism $h \rightarrow \tilde{h}$ of the $k$-algebra $H$ such that $\tilde{T}_{r}=T_{r}\left(r \in S_{0}\right), \tilde{\theta}_{x}=\theta_{x}(x \in P)[3,2.13(\mathrm{c})]$. Note that $\tilde{C}=C$ and $\tilde{C}^{\prime}=C^{\prime}$. Applying this anti-automorphism to the formulas (2) and (3) we get

$$
\begin{aligned}
C \theta_{\rho^{-1}} C^{\prime} & =q^{-\frac{\nu}{2}} \sum_{I \subseteq R^{+}}(-q)^{|I|} \theta_{\rho^{-1}} \theta_{\alpha_{I}} C^{\prime}, \\
C \theta_{\rho} C^{\prime} & =q^{\frac{\nu}{2}} \sum_{I \subseteq R^{+}}(-q)^{-|I|} \theta_{\rho} \theta_{\alpha_{I}^{-1}} C^{\prime} .
\end{aligned}
$$

(For a K-theoretic understanding of formula (44) see [13, 2.6], note that the $C^{\prime}$ here is the $C$ in loc.cit.)

\subsection{The center of $H$}

We have

(a) The center $Z(H)$ of $H$ consists of $W_{0}$-invariant elements in $\Theta$, i.e. $Z(H)$ $=\Theta^{W_{0}}($ see [4] $)$.

Therefore the center $Z(H)$ of $H$ is isomorphic to $k \otimes_{\mathbb{Z}} R_{G}$, where $G$ is a simply connected simple algebraic group over $k$ with root system $R$ and $R_{G}$ is the representation $\operatorname{ring}$ of $G$.

For $w \in W_{0}$ define

$$
e_{w}=w\left(\prod_{\substack{\alpha \in \Delta \\ w(\alpha) \in R^{-}}} x_{\alpha}\right)
$$


(Recall that here $x_{\alpha}$ is the fundamental dominant weight corresponding to $\alpha \in \Delta$.) Then (see [7])

(b) $\Theta$ is a free $Z(H)$-module with a basis $\left\{\theta_{e_{w}} \mid w \in W_{0}\right\}$.

Hence,

(c) for any $x \in P$, the elements $\theta_{x} \theta_{e_{w}}, w \in W_{0}$, form a $Z(H)$-basis of $\Theta$.

(d) For $A, B \in \Theta$, define

$$
(A, B)=(-1)^{\nu} \theta_{\rho} \prod_{\alpha \in R^{+}}\left(1-\theta_{\alpha}\right)^{-1} \sum_{w \in W_{0}}(-1)^{l(w)} w\left(A B \theta_{\rho}\right) \in Z(H) .
$$

By [3, p.163] there exist $\theta_{u}^{\prime} \in \Theta\left(u \in W_{0}\right)$ such that $\left(\theta_{e_{w}}, \theta_{u}^{\prime}\right)=\delta_{w, u}$ and the elements $\theta_{u}^{\prime}$ form a $Z(H)$-basis of $\Theta$.

3.3. Let $T$ be a maximal torus of $G$ and identify $P$ with the character group $\operatorname{Hom}\left(T, k^{*}\right)$ of $T$. Then $\Theta$ is isomorphic to the group algebra $k[P]$ of $P$. For $t \in T$, we have a $k$-algebra homomorphism $\phi_{t}: \Theta \rightarrow k$ defined by $\theta_{x} \rightarrow x(t)$ for all $x \in P$. It is known that the map $t \rightarrow \varphi_{t}$ defines a bijection between $T$ and the set of $k$-algebra homomorphisms from $\Theta$ to $k$.

Now the center $Z(H)$ of $H$ is a free $\Theta$-module of rank $W_{0}$. Therefore every $k$-algebra homomorphisms from $Z(H)$ to $k$ is the restriction $\left.\phi_{t}\right|_{Z(H)}$ of some algebra homomorphism $\phi_{t}: \Theta \rightarrow k$. Moreover, $\left.\phi_{t}\right|_{Z(H)}=\left.\phi_{s}\right|_{Z(H)}$ if and only if $s$ and $t$ are conjugate by some element of $W_{0}$, since $Z(H)=\Theta^{W_{0}}$. Thus the set of $k$-algebra homomorphisms from $Z(H)$ to $k$ is in one-to-one correspondence with the set of semisimple classes of $G$.

For $t \in T$, let $\bar{t}$ be the semisimple class of $G$ containing $t$ and let $\phi_{\bar{t}}=$ $\left.\phi_{t}\right|_{Z(H)}: Z(H) \rightarrow k$ be the corresponding homomorphism. Then let $\mathcal{Z}_{t}$ be the two-sided ideal of $H$ generated by all $z-\phi_{t}(z), z \in Z(H)$. Define $H_{t}=H / \mathcal{Z}_{t}$. We have $\operatorname{dim} H_{t}=\left|W_{0}\right|^{2}$.

For each simple $H$-module $L$, there exist some $t$ in $T$ such that $Z(H)$ acts on $L$ through the homomorphism $\phi_{t}$. So to study simple modules of $H$ it is enough to study simple modules of the quotient algebras $H_{t}$ for $t \in T$. We shall use the same notations $C_{w}^{\prime}, D_{w}, C, C^{\prime}, \theta_{x}, \ldots$ for their images in $H_{t}$.

Theorem 3.4. Let $t \in T$. The following statements are equivalent.

(a) $C H_{t} C=0 .\left(\right.$ Recall that $C=C_{w_{0}}$ and $C^{\prime}=C_{w_{0}}^{\prime}$.) 
(b) $C H_{t} C^{\prime}=0$.

(c) $C^{\prime} H_{t} C=0$.

(d) $C^{\prime} H_{t^{-1}} C^{\prime}=0$.

(e) For any simple $H_{t}$-module $L$ we have $C L=0$.

(f) For any simple $H_{t^{-1}}$-module $L$ we have $C^{\prime} L=0$.

Proof. There is a unique involutive automorphism $h \rightarrow h^{*}$ of the $k$-algebra $H$ such that $T_{r}^{*}=-q T_{r}^{-1}=q-1-T_{r}\left(r \in S_{0}\right), \theta_{x}^{*}=\theta_{x^{-1}}(x \in P)$ [3, 2.13(d)]. Noting that $C^{*}=(-1)^{l\left(w_{0}\right)} C^{\prime}$, we see that (a) and (d) are equivalent, (e) and (f) are equivalent.

Using the involutive anti-automorphism $h \rightarrow \tilde{h}$ of the $k$-algebra $H$ defined by $\tilde{T}_{r}=T_{r}\left(r \in S_{0}\right), \tilde{\theta_{x}}=\theta_{x}(x \in P)[3,2.13(\mathrm{c})]$ and noting that $\tilde{C}=C$ and $\tilde{C}^{\prime}=C^{\prime}$, we see that (b) and (c) are equivalent.

Since the two-sided ideal $H_{c_{0}}$ of $H$ spanned by all $C_{w}, w \in c_{0}$ is generated by $C$, using $[11,7.7]$ we know that (a) and (e) are equivalent.

Now we show that (a) and (b) are equivalent. Since $T_{w} C=C T_{w}=$ $q^{l(w)} C$ if $w \in W_{0}$, we see that $C H C$ is spanned by $C \theta_{x} C$. By formula (II) in 3.1 , we have

$$
C \theta_{x} C=q^{-\frac{l\left(w_{0}\right)}{2}} \sum_{w \in W_{0}} w\left(\theta_{x} \prod_{\alpha \in R^{+}} \frac{1-q \theta_{\alpha}}{1-\theta_{\alpha}}\right) C .
$$

So we have the following assertion.

(i) The condition $\mathrm{CH}_{t} \mathrm{C}=0$ is equivalent to

$$
\phi_{t}\left(\sum_{w \in W_{0}} w\left(\theta_{x} \prod_{\alpha \in R^{+}} \frac{1-q \theta_{\alpha}}{1-\theta_{\alpha}}\right)\right)=0, \quad \text { for all } x \in P
$$

Using 3.2 (c) we know that $H$ is spanned by all $T_{w} z \theta_{\rho} \theta_{e_{u}}, w, u \in W_{0}, z \in$ $Z(H)$. Therefore we have the claim below.

(ii) The condition $\mathrm{CH}_{t} \mathrm{C}=0$ is equivalent to

$$
\phi_{t}\left(\sum_{w \in W_{0}} w\left(\theta_{\rho} \theta_{e_{u}} \prod_{\alpha \in R^{+}} \frac{1-q \theta_{\alpha}}{1-\theta_{\alpha}}\right)\right)=0, \quad \text { for all } u \in W_{0} .
$$


Similar to [10, Lemma 2.10], we see that $H C^{\prime}$ is spanned by all $T_{w} z \theta_{I} C^{\prime}$, $w \in W_{0}, z \in Z(H), I \subseteq \Delta$, where $\theta_{I}=\prod_{\alpha \in I} \theta_{x_{\alpha}}$. Since $T_{w} C=C T_{w}=$ $q^{l(w)} C$ if $w \in W_{0}$ and $C \theta_{I} C^{\prime}=0$ if $I \neq \Delta$, as a $Z(H)$-module, $C H C^{\prime}$ is generated by $C \theta_{\rho} C^{\prime}$.

Recall the formula (51) in 3.1:

$$
C \theta_{\rho} C^{\prime}=q^{\frac{\nu}{2}} \sum_{I \subseteq R^{+}}(-q)^{-|I|} \theta_{\rho} \theta_{\alpha_{I}^{-1}} C^{\prime},
$$

where $\nu=l\left(w_{0}\right)=\left|R^{+}\right|, \alpha_{I}$ is the sum of all roots in $I$ and $|I|$ is the cardinality of $I$.

Let $A=q^{\frac{\nu}{2}} \sum_{I \subseteq R^{+}}(-q)^{-|I|} \theta_{\rho} \theta_{\alpha_{I}^{-1}}$. Note that

$$
A=(-1)^{\nu} q^{-\frac{\nu}{2}} \theta_{\rho}^{-1} \prod_{\alpha \in R^{+}}\left(1-q \theta_{\alpha}\right) .
$$

Thus

$$
\left(A, \theta_{e_{u}}\right)=(-1)^{\nu} q^{-\frac{\nu}{2}} \sum_{w \in W_{0}} w\left(\theta_{\rho e_{u}} \prod_{\alpha \in R^{+}} \frac{1-q \theta_{\alpha}}{1-\theta_{\alpha}}\right) .
$$

Since $A=\sum_{u \in W_{0}}\left(A, \theta_{e_{u}}\right) \theta_{u}^{\prime}$ in $H$ and $\theta_{u}^{\prime} C^{\prime}, u \in W_{0}$, are linearly independent in $H_{t} C^{\prime}$, we obtain the following equivalence condition.

(iii) The condition $\mathrm{CH}_{t} \mathrm{C}^{\prime}=0$ is equivalent to

$$
\phi_{t}\left(\sum_{w \in W_{0}} w\left(\theta_{\rho e_{u}} \prod_{\alpha \in R^{+}} \frac{1-q \theta_{\alpha}}{1-\theta_{\alpha}}\right)=0 \quad \text { for all } u \in W_{0} .\right.
$$

Using (ii) and (iii) we see that (a) and (b) are equivalent. The theorem is proved.

Theorem 3.5. Let $t \in T$ be such that $\alpha(t)=q$ for all simple roots $\alpha$ of $R$. Then

(a) $\mathrm{CH}_{t} \mathrm{C}^{\prime}$ (resp. $\mathrm{C}^{\prime} \mathrm{H}_{t} \mathrm{C}$ ) is a two-sided ideal of $H_{t}$ with dimension 1 if $\sum_{w \in W_{0}} q^{l(w)} \neq 0$.

(b) $C H_{t} C^{\prime}=0$ if $\sum_{w \in W_{0}} q^{l(w)}=0$.

Proof. We have seen that $C H_{t} C^{\prime}$ is spanned by the image in $H_{t}$ of $C \theta_{\rho} C^{\prime}$. To see it is a two-sided ideal of $H_{t}$ it suffices to prove that the images in $H_{t}$ 
of $\theta_{x} C \theta_{\rho} C^{\prime}$ and $C \theta_{\rho} C^{\prime} \theta_{x}$ for all $x \in \Theta$ are scalar multiples of the image of $C \theta_{\rho} C^{\prime}$ in $H_{t}$.

(i) If $w$ is not the neutral element of $W_{0}$, then there exists a positive root $\beta$ such that $w(\beta)=\alpha^{-1}$ for some simple root $\alpha$. Thus $w(1-q \beta)(t)=0$.

Let $A$ be as in the proof of Theorem 3.4. Then $\theta_{x} C \theta_{\rho} C^{\prime}=A \theta_{x} C^{\prime}$. Since

$$
\left(A \theta_{x}, \theta_{e_{u}}\right)=(-1)^{\nu} q^{-\frac{\nu}{2}} \sum_{w \in W_{0}} w\left(\theta_{\rho x e_{u}} \prod_{\alpha \in R^{+}} \frac{1-q \theta_{\alpha}}{1-\theta_{\alpha}}\right)
$$

using (i) we get

$$
\left(A \theta_{x}, \theta_{e_{u}}\right)(t)=\rho(t) x(t) e_{u}(t) \prod_{a \in R^{+}} \frac{1-q^{1+\left\langle\rho, \alpha^{\vee}\right\rangle}}{1-q^{\left\langle\rho, \alpha^{\vee}\right\rangle}},
$$

if $1-q^{\left\langle\rho, \alpha^{\vee}\right\rangle} \neq 0$ for all positive roots $\alpha$. We have (see for example [6, Corollary 2.17])

$$
\prod_{a \in R^{+}} \frac{1-q^{1+\left\langle\rho, \alpha^{\vee}\right\rangle}}{1-q^{\left\langle\rho, \alpha^{\vee}\right\rangle}}=\sum_{w \in W_{0}} q^{l(w)},
$$

if $1-q^{\left\langle\rho, \alpha^{\vee}\right\rangle} \neq 0$ for all positive roots $\alpha$. Now $\left(A \theta_{x}, \theta_{e_{u}}\right)$ is in $Z(H)$, so $\left(A \theta_{x}, \theta_{e_{w}}\right)(t)$ is a regular function in $q \in k^{*}$. Thus we have

$$
\left(A \theta_{x}, \theta_{e_{u}}\right)(t)=(-1)^{\nu} q^{-\frac{\nu}{2}} \rho(t) x(t) e_{u}(t) \sum_{w \in W_{0}} q^{l(w)}
$$

for all $q \in k^{*}$. So the images in $H_{t}$ of $\theta_{x} C \theta_{\rho} C^{\prime}$ for all $x \in \Theta$ are scalar multiples of the image in $H_{t}$ of $C \theta_{\rho} C^{\prime}$, and $C H_{t} C^{\prime}$ is a left ideal of $H_{t}$. Using the involutions $h \rightarrow h^{*}$ and $h \rightarrow \tilde{h}$ of $H$ several times we see that $\mathrm{CH}_{t} \mathrm{C}^{\prime}$ is a left ideal of $H_{t}$ implies that it is also a right ideal of $H_{t}$.

The formula (7) also indicates that $C H_{t} C^{\prime}=0$ if and only if $\sum_{w \in W_{0}} q^{l(w)}$ $=0$. The theorem is proved.

It is easy to check that $T_{s} C H_{t} C^{\prime}=q H_{t} C^{\prime}$ and $C H_{t} C^{\prime} T_{s}=-C H_{t} C^{\prime}$ for all simple reflections $s$ if $\alpha(t)=q$ for all simple roots $\alpha$. So the ideals $C H_{t} C^{\prime}$ and $C^{\prime} H_{t} C$ give natural realizations of some one dimensional representations of $H_{q}$. 


\section{Irreducible Modules Attached to the Lowest Two-Sided Cell}

The main result of this section is the following.

Theorem 4.1. Let $t \in T$, then

(a) The element $C \theta_{\rho} C^{\prime}$ in $H_{t}$ generates an irreducible module $L_{t}$ of $H$ if it is nonzero. Moreover, $C L_{t} \neq 0$ in this case.

(b) The element $C^{\prime} \theta_{\rho} C$ in $H_{t}$ generates an irreducible module $L_{t}^{\prime}$ of $H$ if it is nonzero. Moreover, $C^{\prime} L_{t} \neq 0$ in this case.

Proof. Let $J_{c_{0}}$ be the based ring of $c_{0}$. According to [9], $J_{c_{0}}$ is isomorphic to a $\left|W_{0}\right| \times\left|W_{0}\right|$ matrix ring over $R_{G}$. Let $\mathbf{J}_{c_{0}}=\mathbb{C} \otimes J_{c_{0}}$. Then up to isomorphism, irreducible $\mathbf{J}_{c_{0}}$-modules are naturally in one-to-one correspondence with the semisimple classes of $G$. For the semisimple class containing $t$, let $E_{t}$ be a corresponidng simple $\mathbf{J}_{c_{0}}$-module.

Let $\varphi_{0}: H \rightarrow \mathbf{J}_{c_{0}}$ be Lusztig's homomorphism defined through the basis $C_{w}, w \in W$. Then $E_{t}$ is endowed with an $H$-module structure through the homomorphism. Denote the $H$-module structure on $E_{t}$ by $E_{t, \varphi_{0}}$. We have (see for example the proof of Theorem 3.5 in [13]) the following assertion.

(i) $E_{t, \varphi_{0}}$ is isomorphic to $H_{t} C$.

According to [11, 7.7] and [12, Lemma 2.5], $H_{t} C$ has a simple constituent $L$ such that $C L \neq 0$ if and only if $C H_{t} C \neq 0$. In this case, $L$ is the unique simple constituent of $H_{t} C$ such that $C L \neq 0$ and $L$ is also the unique simple quotient module of $H_{t} C$, i.e., the head of $H_{t} C$.

By Theorem 3.4, $C H_{t} C \neq 0$ is equivalent to $C H_{t} C^{\prime} \neq 0$. Now assume that $C H_{t} C^{\prime} \neq 0$. By the proof of [12, Lemma 2.5], the set

$$
M_{t, 0}=\left\{h \in H_{t} C \mid C_{w} h=0, \quad \forall w \in c_{0}\right\}
$$

is the unique maximal submodule of $H_{t} C$.

We have a natural $H$-module homomorphism: $H_{t} C \rightarrow H_{t} C \theta_{\rho} C^{\prime}, h \rightarrow$ $h \theta_{\rho} C^{\prime}$. Therefore, to prove that $C \theta_{\rho} C^{\prime}$ in $H_{t}$ generates an irreducible module $L_{t}$ of $H$ it suffices to prove that

$$
h \in M_{t, 0} \Longleftrightarrow h \theta_{\rho} C^{\prime}=0 .
$$


Let $\Theta_{t}$ be the image of $\Theta$ in $H_{t}$. Then $H_{t} C$ consists of $\theta C, \theta \in \Theta_{t}$. Since $\Theta$ is a free $Z(H)$-module with a basis $\left\{e_{w} \mid w \in W_{0}\right\}$, and for any $w \in c_{0}$ there exists $\xi, \eta \in \Theta$ such that $C_{w}=\xi C \eta$, we see that $\theta C \in H_{t} C$ is in $M_{t, 0}$ if and only if $C \theta_{e_{w}} \theta C=0$ in $H_{t}$ for all $w \in W_{0}$. By formula (11) in 3.1, this is equivalent to

$$
\phi_{t}\left(\sum_{w \in W_{0}} w\left(\theta_{e_{u}} \theta \prod_{\alpha \in R^{+}} \frac{1-q \theta_{\alpha}}{1-\theta_{\alpha}}\right)\right)=0, \quad \text { for all } u \in W_{0} .
$$

Let $A$ be as in the proof of Theorem 3.4, then

$$
\theta C \theta_{\rho} C^{\prime}=\theta A C^{\prime}
$$

Clearly, $\theta C \theta_{\rho} C^{\prime}=0$ in $H_{t}$ if and only if $\theta_{\rho}^{-1} \theta C \theta_{\rho} C^{\prime}=0$ in $H_{t}$. Since

$$
\theta_{\rho}^{-1} \theta C \theta_{\rho} C^{\prime}=\sum_{u \in W_{0}}\left(\theta_{\rho}^{-1} \theta A, \theta_{e_{u}}\right) \theta_{u}^{\prime} C^{\prime}
$$

and $\theta_{u}^{\prime} C^{\prime}, u \in W_{0}$, are linearly independent in $H_{t} C^{\prime}$, we see that $\theta C \theta_{\rho} C^{\prime}=0$ if and only if $\phi_{t}\left(\left(\theta_{\rho}^{-1} \theta A, \theta_{e_{u}}\right)\right)=0$ for all $u \in W_{0}$. By the formula (6) established in the proof of Theorem 3.4, we have

$$
\left(\theta_{\rho}^{-1} \theta A, \theta_{e_{u}}\right)=(-1)^{\nu} q^{-\frac{\nu}{2}} \sum_{w \in W_{0}} w\left(\theta_{e_{u}} \theta \prod_{\alpha \in R^{+}} \frac{1-q \theta_{\alpha}}{1-\theta_{\alpha}}\right) .
$$

Hence the condition $\phi_{t}\left(\left(\theta_{\rho}^{-1} \theta A, \theta_{e_{u}}\right)\right)=0$ for all $u \in W_{0}$ is exactly the condition (8). We proved (a).

Since $C^{\prime} \theta_{\rho^{-1}} C=(-1)^{l\left(w_{0}\right)} C^{\prime} \theta_{\rho} C$, (b) follows from (a) by applying the involution $h \rightarrow h^{*}$. The theorem is proved.

4.2. The left ideal $\mathcal{I}$ defined in 2.1 is in the kernel of the $H$-module homomorphism $\psi: H \rightarrow H_{t} C^{\prime}, h \rightarrow h C^{\prime}$. Thus $\psi$ induces an $H$-module homomorphism $N \rightarrow H_{t} C^{\prime}$, denoted again by $\psi$. Denote the image of $C_{w}$ in $H / \mathcal{I}$ by the same notation $C_{w}$. Then $C_{w}, w \in Y_{0}$, form a basis of $N=H / \mathcal{I}$.

For each two-sided cell $c$, let $N^{\leq c}$ be the submodule of $N$ spanned by all $C_{w}, w \in Y_{0}$ and $w \leq_{L} u$ for some $u \in c \cap Y_{0}$. Also let $N^{<c}$ be the submodule of $N$ spanned by all $C_{w}, w \in Y_{0}-Y_{0} \cap c$ and $w \leq_{L} u$ for some $u \in c \cap Y_{0}$. Then $\psi\left(N^{<c}\right)$ and $\psi\left(N^{\leq c}\right)$ are submodules of $H_{t} C^{\prime}$. This gives some natural 
submodules of $H_{t} C^{\prime}$. Theorem 4.1 asserts that $\psi\left(N \leq c_{0}\right)=H_{t} C \theta_{\rho} C^{\prime}$ is either 0 or an irreducible submodule of $H_{t} C^{\prime}$. In general, $\psi(N \leq c) / \psi\left(N^{<c}\right)$ maybe 0 or reducible, but it is not clear whether this module is semisimple if it is not 0 . (We refer to [2] for the definition of preorder $\leq_{L}$.)

When $k$ is the field of complex numbers, by [11, Theorem 7.8], $\psi\left(N \leq c_{0}\right)=$ $H_{t} C \theta_{\rho} C^{\prime}$ is 0 if and only if the set

$$
\mathbf{g}_{t, q}=\{X \in \operatorname{Lie}(G) \mid \operatorname{Ad}(t)(X)=q X\}
$$

contains nonzero semisimple elements.

For a simple $H$-module $L$, there exists a unique two-sided cell $c$ of $W$ such that $C_{w} L \neq 0$ for some $w \in c$ and $C_{u} L=0$ for any $u \in W-c$ with $u \leq_{L R} w$. The two-sided cell $c$ is denoted by $c_{L}$ and is called the two-sided cell attached to $L$. (We refer to [2] for the definition of preorder $\leq_{L R}$.)

We have seen that for a simple $H$-module $L, c_{L}=c_{0}$ if and only if $C L \neq$ 0 . Theorem 4.1 gives a computable (in principle) realization of irreducible $H$-modules with attached two-sided cell $c_{0}$.

\section{Acknowledgment}

The author thanks the referee for helpful comments.

\section{References}

1. S. Arkhipov and R. Bezrukavnikov, Perverse sheaves on affine flags and Langlands dual groups, Israel J. Math., 170 (2009), 135-183.

2. D. Kazhdan and G. Lusztig, Representations of Coxeter groups and Hecke algebras, Invent. Math., 53 (1979), 165-184.

3. D. Kazhdan and G. Lusztig, Proof of the Deligne-Langlands conjecture for Hecke algebras, Invent. Math., 87 (1987), 153-215.

4. G. Lusztig, Singularities, character formulas, and a $q$-analog of weight multiplicities, Astérisque 101-102 (1983), 208-227.

5. G. Lusztig, Bases in equivariant K-theory, Represent. Theory 2 (1998), 298-369 (electronic).

6. K. Nelsen and A. Ram, Kostka-Foulkes polynomials and Macdonald spherical functions, Surveys in Combinatorics 2003, C. Wensley ed., London Math. Soc. Lect. Notes 307, Cambridge University Press, 2003, 325-370. 
7. R. Steinberg, On a theorem of Pittie, Topology, 14 (1975), 173-177.

8. N. Xi, An approach to the connectedness of the left cells in affine Weyl groups, Bull. London Math. Soc., 21 (1989), 577-561.

9. N. Xi, The based ring of the lowest two-sided cell of an affine Weyl group, J. Algebra., 134 (1990), 356-368.

10. N. Xi, The based ring of the lowest two-sided cell of an affine Weyl group, II, Ann. Sci. Éc. Norm. Sup., 27 (1994), 47-61.

11. N. Xi, Representations of affine Hecke algebras, Lecture Notes in Mathematics, 1587. Springer-Verlag, Berlin, 1994.

12. N. Xi, Represntations of affine Hecke algebras and the based rings of affine Weyl groups, Jour. Amer. Math. Soc., 20 (2007), 211-217.

13. N. Xi, The based ring of the lowest two-sided cell of an affine Weyl group, III, Algebras and Representation Theory, 19(2016), no.6, 1467-1475 